\title{
DESE NVOLVIMENTO DE UM MODELO FRACTAL PARA A ESTIMATIVA DA CONDUTIVIDADE HIDRÁULICA DE SOLOS NÃO SATURADOS(1)
}

\author{
Carlos Fuentes ${ }^{(2)}$, Antonio Celso Dantas Antonino(3), André Maciel \\ Netto $^{(3)}$, Carlos Alberto Brayner de Oliveira Lira ${ }^{(3)} \&$ J aime J oaquim \\ da Silva Pereira Cabral(4)
}

\begin{abstract}
RESUMO
Baseado nos conceitos da geometria fractal e nas leis de Laplace e de Poiseuille, foi criado um modelo geral para estimar a condutividade hidráulica de solos não saturados, utilizando a curva de retenção da água no solo, conforme representada por um modelo em potência. Considerando o fato de que este novo modelo da condutividade hidráulica introduz um parâmetro de interpolação ainda desconhecido, e que, por sua vez, depende das propriedades dos solos, a validação do modelo foi realizada, utilizando dois valores-limite fisicamente representativos. Para a aplicação do modelo, os parâmetros de forma da curva de retenção da água no solo foram escolhi dos de manei ra a se obter o modelo de van Genuchten. Com a finalidade de obter fórmulas al gébricas da condutividade hidráulica, foram impostas relações entre seus parâmetros de forma. A comparação dos resultados obtidos com o modelo da condutividade e a curva experimental da condutividade dos dois solos, Latossolo Vermelho-Amarelo e Argissolo Amarelo, permitiu concluir que o modelo proposto é simples em sua utilização e é capaz de predizer satisfatoriamente a condutividade hidráulica dos solos não saturados.
\end{abstract}

Termos de indexação: condutividade hidráulica, curva de retenção da água no solo, modelo fractal.

\footnotetext{
(1) Recebido para publicação em janeiro de 2003 e aprovado em dezembro de 2004.

(2) Pesquisador do I nstituto Mexicano de Tecnol ogía del Agua - IMTA. Paseo Cuauhnáhuac No. 8532, Col. Progreso. 62550 J iutepec, Morelos. México. E-mail: cfuentes@tlaloc.imta.mx

(3) Professor do Departamento de Energia Nuclear. Universidade Federal de Pernambuco- UFPE. Av. Prof. Luís Freire 1000. CEP 50740-540 Recife (PE). E-mail: acda@ufpe.br.

(4) Professor do Departamento de Engenharia Civil, UFPE. E-mail: jcabral@ufpe.br
} 


\title{
SUMMARY: A FRACTAL MODEL TO ESTIMATE THE UNSATURATED HYDRAULIC CONDUCTIVITY OF SOILS
}

\begin{abstract}
From a conceptual model based on fractal geometry and Laplace's and Poiseuille's laws, a versatileand general fractal model for the hydraulic conductivity to be used in the soils was devel oped. Thesoil-moisture retention curve is derived from a power model. Due to the fact that the proposed model of hydraulic conductivity introduces a still unknown interpolation parameter, which in turn is a function of soil properties, its limiting values were considered for theanalysis. To apply themode in the soil, theform parameters of the soil-moisture retenti on curve were chosen so as to reproduce van Genuchten's equation. In order to obtain a closed-form equation for thehydraulic conductivity, relationships between theform parameters werei mposed. Thecomparison between thepredicted and experimental hydraulic conductivity from two soils, Oxisol and Yellow Utilsol, demonstrated that the proposed mode is flexible and that it can be used to predict the unsaturated hydraulic conductivity of soils with good accuracy.
\end{abstract}

Index terms: hydraulic conductivity, soil-moisture retention curve, fractal model.

\section{INTRODUÇÃO}

A lei de Darcy é largamente utilizada no estudo do movimento da água no solo em várias situações de interesse, tais como: a irrigação e a drenagem (Braud et al., 1995; Falleiros et al., 1998; Netto et al., 2000). A aplicação desta lei requer, entretanto, o conhecimento das características hidrodinâmicas do solo, ou seja: (a) a relação entre o potencial matricial $(\psi)$ e a umidade volumétrica do solo, $\theta(\psi)$, conhecida como curva característica da umidade do solo ou curva de retenção da água no solo, e (b) a relação entrea condutividade hidráulica ea umidade volumétrica, $K(\theta)$.

Para avaliar as características hidrodinâmi cas do solo, têm sido propostos diferentes métodos, tanto de laboratório (Klute \& Dirksen, 1986; van Dam et al., 1994) como de campo (Hillel et al., 1972; Libardi et al., 1980; Angulo-J aramillo et al., 2000). A curva de retenção pode ser determinada por meio de métodos indiretos, utilizando al gumas propriedades do solo, como a textura, a porosidade e a matéria orgânica (Gupta \& Larson, 1979; Rawls \& Brakensiek, 1981; Haverkamp \& Parlange, 1986; Saxton et al., 1986). A condutividade hidráulica é, em geral, estimada a partir de model os da curva de retenção da água no solo (Burdine, 1953; Mualem, 1976; Zhang \& van Genuchten, 1994). Nestes métodos, a curva de retenção é descrita por fórmulas algébricas, as quais são introduzidas nos modelos de predição da condutividade hidráulica (Brooks \& Corey, 1964; van Genuchten, 1980).

Os modelos de predição de $K(\theta)$ estão baseados nas leis de Laplace e de Poiseuille, as quais relacionam, respectivamente, a pressão e a velocidade média da água nos poros com o tamanho do poro. Fazendo al gumas considerações teóricas, o fluxo de Darcy é obtido pela integração dos fluxos deágua em todos os poros. Os model os sãovalidados e, ou, corrigidos mediante a comparação dos resultados teóricos com as curvas experimentais em solos selecionados (Childs \& Collis-George, 1950; Burdine, 1953; Mualem, 1976; Fuentes, 1992).

Recentemente, Fuentes et al. (2001) utilizaram al guns conceitos da geometria fractal, no intuito de justificar as correções empíricas empregadas nos modelos clássicos da condutividade hidráulica, e propuseram esta abordagem fractal para a unificação dos modelos. Este modelo geral tem permitido obter novos modelos particulares da condutividade, quando são introduzidas hipóteses adequadas de definição do tamanho de poro.

O objetivo do presente trabalho foi deduzir um model o fractal de predição da condutividade hidráulica dos solos não saturados, utilizando uma nova formulação para a distribuição bidimensional dos tamanhos de poro e demonstrar sua validade, aplicando este model o a dois ti pos distintos de solos.

\section{MATERIAL E MÉTODOS}

Nesta seção, serão apresentados os principais conceitos, definições e hipóteses que possibilitaram a obtenção do modelo. Em analogia direta com o termo porosidade, que se refere à parte porosa do meio, o termo solidosidade será introduzido para descrever a fração sólida deste meio. O termo areal será utilizado para descrever grandezas relativas à área do mesmo modo que a palavra volumétrica refere-se a volume. As várias passagens que requerem maior desenvol vimento matemático estão detal hadas no apêndice.

O model o da condutividade hidráulica, proposto por Fuentes et al. (2001), fundamenta-se na lei de Poiseuille, quando o fenômeno é observado na escala dos poros. Utilizando a distribuição bidimensional 
dos tamanhos de poro, $\mathrm{R}_{\mathrm{s}}$, a integração desta lei sobre o domínio dos poros que contêm água, em uma seção do solo perpendicular à direção do movimento, permite obter a vazão por unidade de superfície ou fluxo de Darcy, fornecendo o seguinte resultado:

$$
\mathrm{K}=\mathrm{C}_{\mathrm{f}} \frac{\rho_{\mathrm{W}} \mathrm{g}}{\eta} \int_{\tilde{\Omega}} R_{\mathrm{S}}^{2} \mathrm{~d} \omega
$$

sendo $\rho_{\mathrm{w}}$ a massa específica da água, $\eta$ o coeficiente de viscosidade dinâmica da água, g a aceleração gravitacional, $\mathrm{C}_{\mathrm{f}}$ ocoeficiente de Koseny (Bear, 1972) que para um poro circular vale $C_{f}=1 / 8, \tilde{\Omega}$ o domínio areal dos poros cheios com água, $\mathrm{d} \omega=\mathrm{dA} / \mathrm{A}_{\mathrm{T}}$ é uma fração da área $(A)$ destes poros, relativa à área total da seção do solo $\left(A_{T}\right)$, e $K$ é a condutividade hidráulica.

A definição da distribuição de $R_{s}$ requer a introdução do conceito de tortuosidade das trajetórias de fluxo no meio poroso. A tortuosidade T é definida como (Figura 1):

$$
\mathrm{T}=\frac{\mathrm{dz}_{\mathrm{f}}}{\mathrm{dz}} \geq 1
$$

sendo $z$ a coordenada que descreve a trajetória do movimento na direção macroscópica e $z_{\mathrm{f}}$ representa a trajetória "real" que segue a água no meio poroso (Dullien, 1979).

As vel oci dades sobre a trajetória retilínea e sobre a trajetória tortuosa estão representadas, respectivamente, por $\mathrm{v}=\mathrm{dz} / \mathrm{dt} \mathrm{ev}_{\mathrm{f}}=\mathrm{dz} / \mathrm{dt}$. Deacordo com o que está apresentado na figura 1, infere-se que:

$$
T=\frac{v_{f}}{v}=\frac{R}{R_{S}}
$$

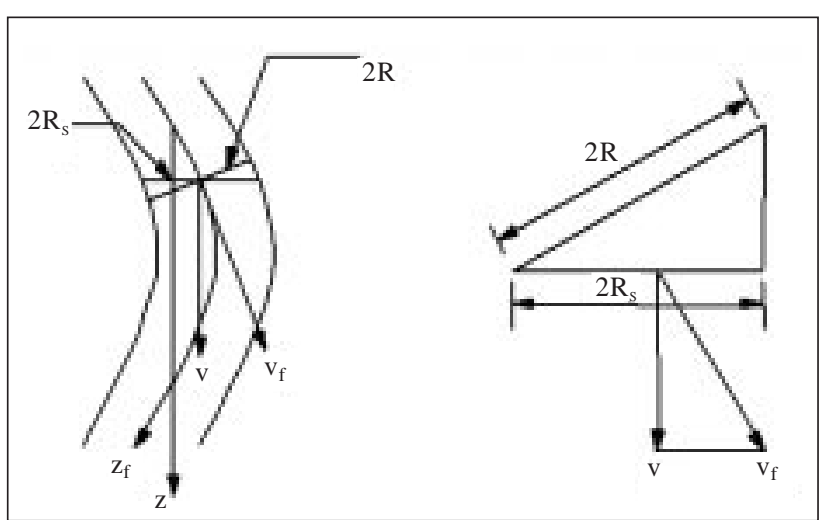

Figura 1. A tortuosidadee os triângul os semelhantes formados pelos raios e as velocidades. A velocidade $\mathrm{v}$ é referenciada à trajetória $\mathrm{z}$ do movimento e a velocidade $\mathrm{v}_{\mathrm{f}}$ é referenciada à trajetória "tortuosa" do movimento.
O raio capilar ( $\mathrm{R}$ ) está relacionado com o potencial matricial da água no solo $(\psi)$, de acordo com a lei de Laplace:

$$
\mathrm{R}=-\frac{2 \sigma \cos \left(\alpha_{\mathrm{c}}\right)}{\rho_{\mathrm{W}} \mathrm{g} \psi}
$$

sendo $\sigma$ a tensão superficial na interface água-ar e $\alpha_{c}$ o ângulo de contato formado por esta interface com as partículas do solo.

É importante observar quea variável $\omega$, conforme utilizada na equação 1, é uma medida da área perpendicular à direção macroscópica exposta pelos poros, ou seja, $\omega$ está relacionada com $R_{s}$, enquanto a medida do volume dos poros (V) relativa ao vol ume total de solos $\left(\mathrm{V}_{\mathrm{T}}\right)$, denotada por $\varepsilon$ e definida de maneira que $\mathrm{d} \varepsilon=\mathrm{dV} N_{\mathrm{T}}$, está relacionada com o raio perpendicular à trajetória tortuosa (R). A área total dos poros expostos, relativa à área total do corte perpendicular do solo, ou porosidade areal total $(\mu)$, pode ser obtida por:

$$
\widetilde{\Omega}_{\mathrm{T}}^{\int \mathrm{d} \omega=\mu}
$$

na qual $\tilde{\Omega}_{\mathrm{T}}$ representa o domínio areal total dos poros. O volume total dos poros relativo ao volume total do solo considerado, ou porosidade volumétrica total $(\phi)$, pode ser obtido com a seguinte integração:

$$
\int_{\Omega_{\mathrm{T}}} \mathrm{d} \varepsilon=\varphi
$$

na qual $\Omega_{\top}$ representa o domínio volumétrico total dos poros. Quando o meio poroso está totalmente preenchido com água, a porosidade volumétrica é igual à umidade volumétrica $(\theta)$.

É de interesse fundamental conhecer a dependência entrea condutividade hidráulica (K) e a umidade volumétrica $(\theta)$, a partir da relação $\theta(\psi)$. Para estabelecer a relação entre ambas as curvas, é necessário primeiramente determinar a relação entre os raios $\mathrm{R}$ e $\mathrm{R}_{\mathrm{s}}$, ou seja, deve-se encontrar a tortuosidade de cada capilar e a relação entre as porosidades $\varepsilon$ e $\omega$. Fuentes et al. (2001) basearamse na idéia probabilística introduzida por Childs \& Collis-George (1950) para encontrar esta relação, conforme descrito abaixo.

Fazendo um corte perpendicular à trajetória macroscópica do fluxo, obtêm-sedois planos, os quais incluem as seções S' e S" (Figura 2). Os raios dos poros na seção $S^{\prime}$ são denotados por $\left\{r, r_{s}\right\}$ e os da face na seção $S^{\prime \prime}$ por $\left\{\xi, \xi_{s}\right\}$. Uma partícula de água situada em um poro do plano $S^{\prime}$ pode continuar sua trajetória pelo mesmo poro capilar ou trocar por outro poro de diferente tamanho. A modelagem destas possibilidades de troca pode ser feita com a introdução da probabilidade do encontro dos planos em uma seção intermediária virtual S*. 


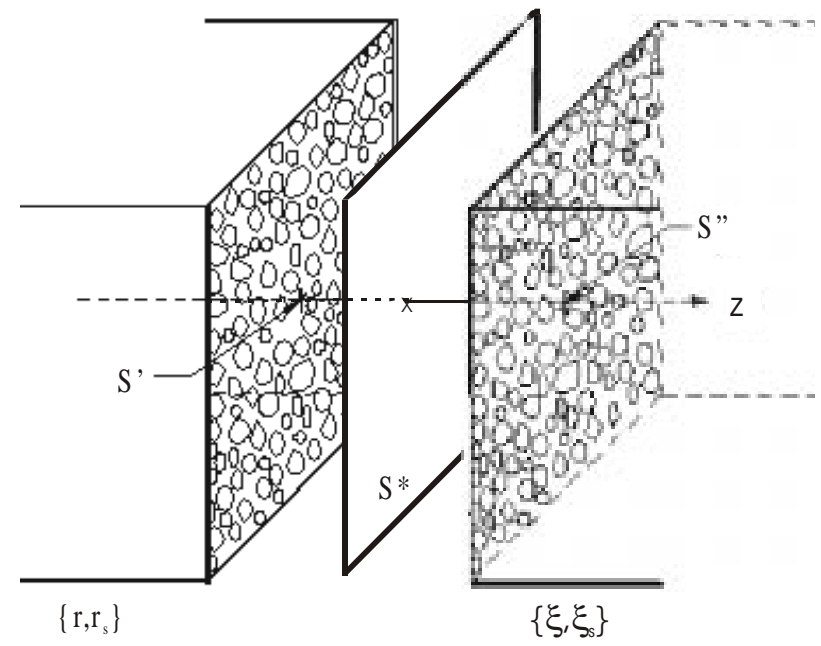

Figura 2. Um corte no solo perpendicular à direção macroscópica do fluxo. Os raios dos poros são caracterizados em cada face por $\left\{r, r_{s}\right\}$ e $\left\{\xi, \xi_{s}\right\}$.

Childs \& Collis-George (1950) não fizeram distinção entre os raios $R$ e $R_{s}$ e consideraram quea função densidade da porosidade volumétrica $f(r)$ éa mesma nas duas seções e iguais à função densidade do fluxo areal em cada seção. A probabilidade do interval o que contém r é precisamenteigual à função de densidade do fluxo areal em cada seção. A probabilidade do intervalo que contém $r$ sobre a outra seção é igual a $d \varepsilon(\xi)=f(\xi) d \xi$. A probabilidade de que os poros representados por estes intervalos encontrem-se de uma maneira completamente al eatória em uma seção S* intermediária entre S' e $S^{\prime \prime}$ é o produto das duas probabilidades. O produto das áreas elementares $d \varepsilon(r)$ e $d \varepsilon(\xi)$ representa a área comum do fluxo: $d \omega(r, \xi)=d \varepsilon(r) d \varepsilon(\xi)=f(r) d r f(\xi) d \xi$. A integração desta equação sobre toda a classe de raios de poros leva a: $\mu=\phi \phi=\phi^{2}$.

No modelo de Purcell (1949), o sistema está representado por capilares paral el os em que o fator de tortuosidade é independente do raio de poro e a partícula deágua continua pelo mesmo poro, ou seja, trata-se de evento total mente correlacionado. Neste caso, éfácil deduzir quea porosidade areal éigual a porosidade volumétrica: $d \omega(r)=d \varepsilon(r)=f(r) d r$ e em tal situação $\mu=\phi$.

Em termos probabilísticos, o modelo de Purcell representa uma correlação compl eta entre as duas seções, ao passo que o modelo de Childs \& CollisGeorge representa uma decorrelação completa. Mualem \& Dagan (1978) assinalaram que o modelo de Purcell para a estimativa da condutividade hidráulica pode ser formal mente deduzi do do modelo de Childs \& Coll is-George, sea área comum do fluxo for redefinida como: $d \omega(r, \xi)=f(r) d r \delta(\xi-r) d \xi$, na qual $\delta$ é o delta de Dirac. É de se esperar que o comportamento real da água no solo possa ser descrito por um modelo intermediário entre aquele de Purcell e o de Childs \& Collis-George, pois, geral mente, o fluxo de água no solo não ocorre como em um sistema de capilares paralelos, tampouco é completamente aleatório, já que aparentemente existe uma estrutura hierarquizada no solo.

Nopresentetrabalho, admite-sea hipótese deque o solo possa ser considerado um objeto fractal (Mandelbrot, 1983; Rieu \& Sposito, 1991a,b; Oleschko et al., 1997; Fuentes et al., 1998, 2001), e demonstra-se então que este comportamento intermediário, e possivel mente mais realista, pode ser obtido. O fundamento básico para esta dedução é a relação entre a área e o volume de Mandelbrot.

Na geometria de Euclides, como a dimensão do volume $(V)$ de um objeto é $L^{3}$ e de sua área é $L^{2}$, tem-se que $V^{1 / 3}$ é proporcional a $A^{1 / 2}$. Deacordo com Mandel brot, na geometria fractal, $V^{1 / 3}$ é proporcional a $A^{1 / D}$, sendo $D$ a dimensão fractal do objeto. Esta proporção entre superfície e volume pode ser generalizada da seguinte maneira: $\sup _{E}(F) \propto$ $\left[\mathrm{Vol}_{\mathrm{E}}(\mathrm{F})\right]^{\mathrm{D} / \mathrm{E}}$, na qual $\mathrm{F}$ é um objeto imerso em um espaço de dimensão euclidiana igual a $E$, e, para os sol os como objetos reais, tem-se que $\mathrm{E}=3$.

Se $\phi_{\mathrm{s}}=1$ - $\phi$ representa o volume dos sólidos relativo ao volume total do solo, ou "solidosidade vol umétrica", então a área dos sólidos relativa à área total do solo, ou "solidosidade areal", $\mu_{\mathrm{s}}$ será igual a $\phi_{\mathrm{s}}^{\mathrm{s}}$, com $\mathrm{S}=\mathrm{D} / \mathrm{E}$. Segundo a idéia probabilística, sobre cada plano a fração da área poral será $\phi^{\text {s. A }}$ probabilidade total do encontro das seções em um ponto intermediário representa a fração da área disponível para o fluxo: $\mu=\phi^{s} \phi^{s}=\phi^{2 s}$.

Posto que $\phi_{\mathrm{s}}+\phi=1$ e $\mu_{\mathrm{s}}+\mu=1$, estabelece-se a relação fundamental seguinte:

$$
(1-\phi)^{\mathrm{s}}+\phi^{2 \mathrm{~s}}=1
$$

É importante observar que, para o desenvolvimento seguinte, a solidosidade areal e a porosidade areal são, respectivamente:

$$
\begin{gathered}
\mu_{\mathrm{s}}=1-\mu=(1-\phi)^{\mathrm{s}}=\phi_{\mathrm{s}}^{\mathrm{s}} \\
\mu=\phi^{\mathrm{s}} \phi^{\mathrm{s}}=\phi^{2 \mathrm{~s}}
\end{gathered}
$$

Quando os poros não se encontram totalmente cheios com água, a relação entre as porosidades (areal e volumétrica), definida pela equação 9, deve ser generalizada às porosidades parciais, a saber:

$$
\omega=\varepsilon^{2 s} \operatorname{com} 0 \leq \omega \leq \mu \text { e } 0 \leq \varepsilon \leq \phi
$$

No trabalho de Brooks \& Corey (1964), argumenta-se experimentalmente que a curva de retenção da água no solo é bem representada pela função de potência $\theta(\psi) \propto 1 /|\psi|^{\lambda}$, quando $|\psi| \gg 0$, na qual $\lambda>0$ é uma potência chamada de "índice de poros". Considerando a lei de Laplace, definida pela equação 4 , deduz-se que $\varepsilon(R) \propto R^{\lambda}$ quando $R \rightarrow 0$. Definindo um tamanho de poro crítico $\left(R_{0}\right)$ associado a uma pressão crítica no sentido de Brooks \& Corey eà porosidade volumétrica total, pode-se escrever a 
porosi dade vol umétrica parcial em função de R como $\varepsilon(R)=\left(R / R_{0}\right)^{\lambda} \phi$. Visto que $R$ é o raio perpendicular à trajetória tortuosa e $\mathrm{R}_{\mathrm{s}}$ é o raio perpendicular à trajetória retilínea em determinado ponto, érazoável supor que a contribuição relativa à porosidade volumétrica do primeiro seja a mesma contribuição relativa à porosidade areal do segundo. Em outras palavras, pode-se escrever $\omega\left(R_{s}\right)=\left(R_{s} / R_{s o}\right)^{\lambda} \mu$, na qual o raio $R_{\text {so }}$ corresponde ao raio $R_{0}$. Tendo em conta o anterior e as equações 9 e 10, obtém-se a relação seguinte entre $R_{s}$ e $R$ :

$$
\frac{\mathrm{R}_{\mathrm{S}}}{\mathrm{R}_{\mathrm{SO}}}=\left(\frac{\mathrm{R}}{\mathrm{R}_{\mathrm{O}}}\right)^{2 \mathrm{~s}}
$$

A tortuosidade em função de cada raio de poro (R) resulta da comparação das equações 3 e 11:

$$
\mathrm{T}(\mathrm{R})=\mathrm{T}_{\mathrm{O}}\left(\frac{\mathrm{R}_{\mathrm{O}}}{\mathrm{R}}\right)^{\delta} \operatorname{com} 0<\delta=2 \mathrm{~s}-1<1
$$

sendo $T_{0}=R_{0} / R_{\text {so }}$.

$\mathrm{Na}$ abordagem fractal, a área elementar comum ao fluxo é expressa por:

$$
d \omega\left(r_{s}, \xi_{s}\right)=d \varepsilon^{s}(r) d \varepsilon^{s}(\xi)
$$

A equação 1 , que define a condutividade hidráulica, toma então a seguinte forma:

$$
\frac{\mathrm{K}}{\mathrm{K}_{\mathrm{S}}}=\frac{\int\left[\mathrm{R}_{\mathrm{S}}\left(\mathrm{r}_{\mathrm{s}}, \xi_{\mathrm{S}}\right)\right]^{2} \mathrm{~d} \varepsilon^{\mathrm{s}}(\mathrm{r}) \mathrm{d} \varepsilon^{\mathrm{s}}(\xi)}{\int_{\Omega_{\mathrm{T}}}\left[\mathrm{R}_{\mathrm{S}}\left(\mathrm{r}_{\mathrm{s}}, \xi_{\mathrm{S}}\right)\right]^{2} \mathrm{~d} \varepsilon^{\mathrm{s}}(\mathrm{r}) \mathrm{d} \varepsilon^{\mathrm{s}}(\xi)}
$$

na qual $K_{s}$ é a condutividade hidráulica em meio saturado e o processo é suposto isotérmico ( $\eta$ e $\rho_{w}$ constantes).

A maneira decal cular a distribuição bidimensional dos tamanhos de poro, $R_{s}$, a partir dos raios $r_{s}$ e $\xi_{s}$, permite obter diversos model os da condutividade hidráulica (Fuentes et al., 2001). O modelo aqui desenvolvido foi baseado na hipótese seguinte:

$$
\mathrm{R}_{\mathrm{S}}=\mathrm{r}_{\mathrm{S}}^{\alpha} \xi_{\mathrm{S}}^{1-\alpha}
$$

sendo $\alpha$ um parâmetro de interpolação dependente das propriedades do solo. Em virtude da natureza simétrica de $R_{s}$ em relação a $r_{s}$ e $\xi_{s}$ proporcionada por este modelo, é suficiente tomar o intervalo $1 / 2 \leq \alpha \leq 1$, verificando que $\alpha=1 / 2$ corresponde ao modelo da média geométrica, e $\alpha=1$ corresponde a um model o de "poro neutro", ou seja, $R_{s}=r_{s}$.

I ntroduzindoa lei de Laplace (equação 4) eas equações 11 e 15 na equação 14, o model o da condutivi dade hidráulica relativa correspondente é o seguinte:

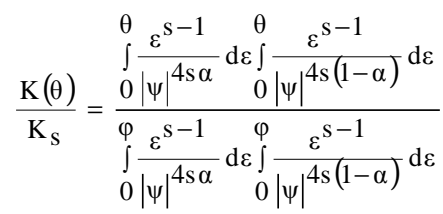

nele podendo ser incorporada a umidade volumétrica de água residual $\left(\theta_{r}\right)$, definida por Brooks \& Corey (1964), de maneira que $K\left(\theta_{r}\right)=0$, trocando $\theta$ por $\theta_{\text {ef }}=$ $\theta-\theta_{\mathrm{r}}$ e $\phi$ pela porosidade volumétrica efetiva $\phi_{\mathrm{ef}}=\theta-\theta_{\mathrm{r}}$. Em condições naturais de campo, a saturação completa raramente será al cançada, pois certa quantidade de ar restará presa nos poros do solo.

Um dos grupos mais abrangentes de modelos encontrados na literatura para o potencial $(\psi)$ é o modelo em função de potência seguinte (Braddock et al., 2001):

$$
\psi=\psi_{\mathrm{d}} \mathrm{S}_{\mathrm{e}}^{-1 / \lambda}\left(1-\mathrm{S}_{\mathrm{e}}^{1 / \mathrm{m}}\right)^{1 / \mathrm{n}}
$$

sendo $\mathrm{S}_{\mathrm{e}}$, o grau de saturação efetiva, definido por:

$$
\mathrm{S}_{\mathrm{e}}=\frac{\theta-\theta_{\mathrm{r}}}{\theta_{\mathrm{S}}-\theta_{\mathrm{r}}}
$$

$\left(\psi_{d}\right)$ é um valor característico da pressão; $m>0, n>$ e $\lambda>0$ são três parâmetros de forma e $\left(\theta_{s}\right)$ é a umi dade volumétrica sob saturação natural.

Combinando as equações 16 e 17, adotando $\lambda=\mathrm{mn}$, para reproduzir o model o de van Genuchten, e perfazendo as integrações (Apêndice), chega-se ao model o da Média Geométrica $(\alpha=1 / 2)$ :

$$
\mathrm{K}\left(\mathrm{S}_{\mathrm{e}}\right)=\mathrm{K}_{\mathrm{S}}\left[1-\left(1-\mathrm{S}_{\mathrm{e}}^{1 / \mathrm{m}}\right)^{1-2 \mathrm{~s} / \mathrm{n}}\right]^{2}, \quad \mathrm{n}=\frac{2 \mathrm{~s}}{1-\mathrm{sm}}
$$

e ao modelo do Poro Neutro $(\alpha=1)$ :

$$
\mathrm{K}\left(\mathrm{S}_{\mathrm{e}}\right)=\mathrm{K}_{\mathrm{S}} \mathrm{S}_{\mathrm{e}}^{5}\left[1-\left(1-\mathrm{S}_{\mathrm{e}}^{1 / \mathrm{m}}\right)^{1-4 \mathrm{~s} / \mathrm{n}}\right], \quad \mathrm{n}=\frac{4 \mathrm{~s}}{1-\mathrm{sm}}
$$

com a imposição de que $0<\mathrm{sm}<1$ em ambos os modelos.

Para avaliar o desempenho dos modelos da Média Geométrica e do Poro N eutro, foram utilizados três critérios estatísticos diferentes: (a) a razão de desvios, RD; (b) a eficiência da modelagem, EM, e (c) o coeficiente de massa residual, CMR, calculados, respectivamente, por Willmott et al. (1985):

$$
\mathrm{RD}=\frac{\sum_{\mathrm{i}=1}^{\mathrm{N}}(\mathrm{Mi}-\overline{\mathrm{M}})^{2}}{\sum_{\mathrm{i}=1}^{\mathrm{N}}(\mathrm{Pi}-\overline{\mathrm{M}})^{2}}
$$

$$
\mathrm{EM}=\left[\frac{\sum_{\mathrm{i}=1}^{\mathrm{N}}(\mathrm{Mi}-\overline{\mathrm{M}})^{2}-\sum_{\mathrm{i}=1}^{\mathrm{N}}(\mathrm{Pi}-\mathrm{Mi})^{2}}{\sum_{\mathrm{i}=1}^{\mathrm{N}}(\mathrm{Mi}-\overline{\mathrm{M}})^{2}}\right]
$$

$$
\mathrm{CMR}=\left[\frac{\sum_{\mathrm{i}=1}^{\mathrm{N}} \mathrm{Mi}-\sum_{\mathrm{i}=1}^{\mathrm{N}} \mathrm{Pi}}{\sum_{\mathrm{i}=1}^{\mathrm{N}} \mathrm{Mi}}\right]
$$


sendo Pi os valores das condutividades hidráulicas preditas pelos modelos; $\mathrm{Mi}$ e $\overline{\mathrm{M}}$ os valores experimentais emédia dos val ores das condutividades hidráulicas, respectivamente, e N o número de determinações.

A RD descrevea razão entre o espal hamento das determinações experimentais e o espal hamento dos valores calculados pelo modelo. A EM indica se o modelo fornece uma estimativa melhor das determinações experimentais do que o val or médio dessas determinações. Para RD e EM, o valor esperado tende a 1 (um), quando os valores experimentais e aqueles preditos pelo modelo são consistentes. O CMR indica se o modelo tende a superestimar $(C M R<0)$ ou a subestimar $(C M R>0)$ os valores determinados experimentalmente. $\mathrm{Na}$ ausência de desvios sistemáticos entre os valores preditos e experimentais, o val or esperado de CMR tende a zero.

\section{VALIDAÇÃO DO MODELO}

O model o foi aplicado a dois solos: um Latossolo Vermelho-Amarelo e um Argissolo Amarelo. As características físico-hídricas destes solos foram obtidas em trabalhos anteriores, para o Latossolo Vermelho-Amarelo (Reichardt, 1988) e para o Argissol o Amarelo (Netto et al., 2000). No quadro 1, estão apresentados os val ores de $\theta_{s}$ eK $K_{s}$, eos valores des, obtidos com a equação 7 assumindo $\phi=\theta_{s}$, para os dois solos.

Quadro 1. Valores de $\theta_{s}, K_{s}$ e s para o Latossolo Vermelho-Amarelo e para o Argissolo Amarelo

\begin{tabular}{lccc}
\hline \multicolumn{1}{c}{ Solo } & $\boldsymbol{\theta}_{\mathbf{s}}$ & $\mathbf{K}_{\mathbf{s}}$ & $\mathbf{s}$ \\
\hline & $\mathrm{m}^{3} \cdot \mathrm{m}^{-3}$ & $\mathrm{~m} \cdot \mathrm{s}^{-1}$ & \\
Latossolo Vermel ho-Amarelo & 0,385 & $1,83 \cdot 10^{-5}$ & 0,6704 \\
Argisssolo Amarelo & 0,240 & $8,06 \cdot 10^{-5}$ & 0,6398 \\
\hline
\end{tabular}

Embora não se conheça a dependência do parâmetro $(\alpha)$ com respeito às propriedades dos sol os, neste trabalho, será ilustrada a aplicação aos dois casos-limite $(\alpha=1 / 2$ e $\alpha=1)$ que englobam, portanto, todos os valores deste parâmetro que apresentam significadofísico. Em particular, utilizam-sea curva de retenção da água no solo, dada pela equação 17 $\operatorname{com} \lambda=m n$, eas curvas de condutividade hidráulica, dadas pelas equações 19 e 20.

No quadro 2, estão mostrados os valores de $\theta_{r}$, $\left|\psi_{d}\right|$ e m obtidos do ajuste da equação 17 aos dados experimentais, considerando as relações apropriadas entre $\mathrm{m}$ e $\mathrm{n}$, conforme estabelecidas nas equações 19 e 20. Também mostra, por meio do coeficiente de determinação $R^{2}$, a correlação entre os dados experimentais e a explicação proporcionada pel o modelo.

$\mathrm{Na}$ figura 3, estão apresentadas as curvas de retenção da água no solo observadas e ajustadas (equação 17) com a relação entrem en obtida a partir dos modelos da Média Geométrica $(\alpha=1 / 2)$ (equação 19) e do Poro Neutro $(\alpha=1)$ (equação 20) para: (a) Latossolo Vermel ho-Amarelo e (b) Argissolo Amarelo. Na figura 3, também estão apresentadas as comparações entre as curvas da condutividade hidráulica preditas pelos modelos e aquelas observadas para os dois solos.

Segundo Netto et al. (2000), os valores finais de $K(\theta)$ experimentais são afetados pelos erros inerentes à determinação das densidades de fluxos e dos gradientes de potencial. Conforme o método empregado para a determinação da condutividade hidráulica (Método do Perfil I nstantâneo), N etto et al. (2000) destacaram que um erro de $\pm 50 \%$ sobreo gradiente de potencial provoca um valor de condutividade hidráulica final multiplicado ou dividido por dois. U ma vez que o model o versátil da condutividade hidráulica é baseado na geometria fractal e nas leis de Laplacee Poiseuille, a diferença encontrada nas curvas de condutividade hidráulica experimental e predita pelo modelo, principalmente para altos val ores de umi dade, ocorreprovavel mente por erros inerentes na determinação experimental da condutividade hidráulica supradiscutidos.

Quadro 2. Valores dos parâmetros $\theta_{r},\left|\psi_{d}\right|$ e $m$ da curva de retenção para as diferentes relações entre $m$ e n das fórmulas algébricas da condutividade hidráulica e o coeficiente de determi nação

\begin{tabular}{|c|c|c|c|c|}
\hline Solo & $\theta_{\mathbf{r}}$ & $\left|\psi_{\mathbf{d}}\right|$ & $\mathbf{m}$ & $\mathbf{R}^{2}$ \\
\hline & $m^{3} m^{-3}$ & $m^{3} m^{-3}$ & & \\
\hline & \multicolumn{4}{|c|}{ Modelo M édia Geométrica ( $\alpha=1 / 2$ ) } \\
\hline Latossolo Vermel ho-Amarelo & 0,0513 & 0,3405 & 0,2112 & 0,9984 \\
\hline \multirow[t]{2}{*}{ Argissolo Amarelo } & 0,0000 & 0,10937 & 0,2521 & 0,9702 \\
\hline & \multicolumn{4}{|c|}{ Modelo Poro Neutro $(\alpha=1)$} \\
\hline Latossolo Vermel ho-Amarelo & 0,0438 & 0,2194 & 0,0910 & 0,9965 \\
\hline Argissolo Amarelo & 0,0000 & 0,0800 & 0,1209 & 0,9836 \\
\hline
\end{tabular}


A análise estatística entre os valores das condutividades hidráulicas, preditas pelos modelos da Média Geométrica e do Poro Neutro, e os valores experimentais estão apresentados no quadro 3. Observa-se que, para os dois model os e em ambos os solos, os valores de RD e EM estão próximos de um eos de CMR são positivos debaixo módulo, indicando que os model os tendem a subestimar ligeiramente os valores experimentais. Globalmente, como mostra o resultado da análise estatística, não

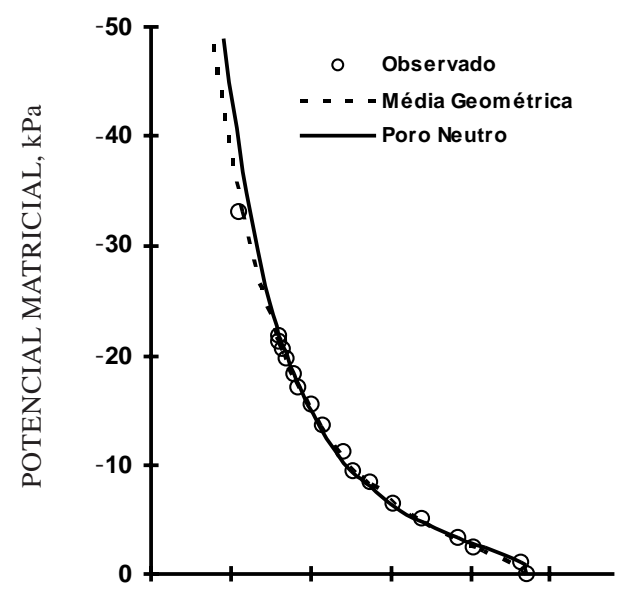

(a)
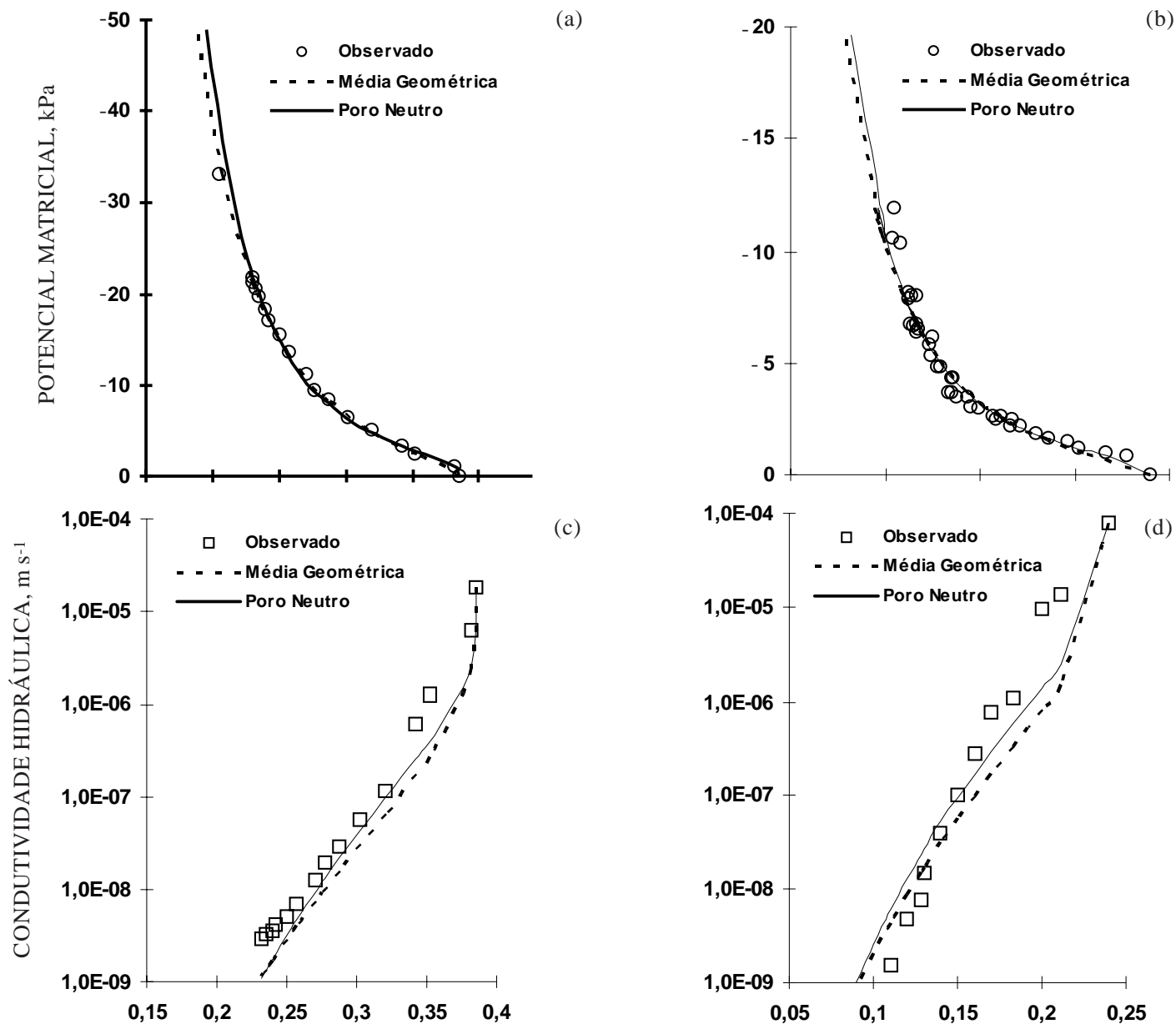

(c)

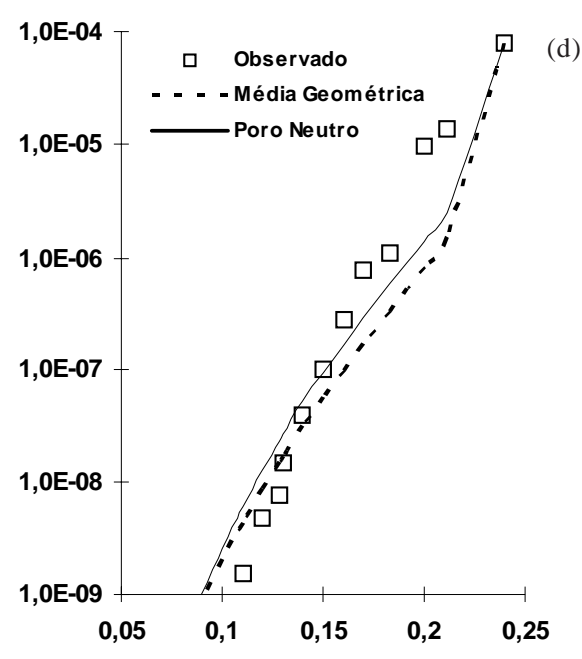

UMIDADE VOLUMÉTRICA, $\mathrm{m}^{3} \mathrm{~m}^{-3}$

Figura 3. Curvas de retenção da água no solo observadas e ajustadas (equação 17) com a relação entre $m$ e n obtida a partir dos modelos da Média Geométrica (equação 19) e do Poro Neutro (equação 20) para: (a) Latossolo Vermelho-Amarelo; (b) Argissolo Amarelo e curvas da condutividade hidráulica preditas pelos modelos para: (c) Latossolo Vermelho-Amarelo e (d) Argissolo Amarelo.

Quadro 3. Resultado da análise estatística entre os valores das condutividades hidráulicas preditas pelos model os da Média Geométri ca e do Poro Neutro e os valores experimentais para o Latossolo Vermel hoAmarelo e o Argissolo Amarelo

\begin{tabular}{lccc}
\hline \multicolumn{1}{c}{ Modelo } & RD & E M & CMR \\
& & Latossolo Vermelho-Amarelo & 0,205 \\
Média Geométrica & 1,055 & 0,949 & 0,193 \\
Poro Neutro & 1,057 & Argissolo Amarelo & 0,210 \\
& & 0,963 & 0,191 \\
Média Geométrica & 0,985 & 0,968 & 0,1989 \\
Poro Neutro & 0,989 & & \\
\hline
\end{tabular}

RD: razão de desvios; EM: eficiência da modelagem e CMR: coeficiente de massa residual. 
existem diferenças significativas na capacidade de descrever os dados experimentais, quando se usam as curvas de retenção da água no sol o correspondentes às diferentes relações entre m e $n$ introduzidas pel os model os da Média Geométrica e do Poro Neutro.

\section{CONCLUSÕES}

1. A partir de um modelo conceitual baseado na geometria fractal enas leis fundamentais de Laplace e Poiseuille, foi criado um modelo versátil para estimar a condutividade hidráulica em solos.

2. Embora este novo model o tenha introduzido o parâmetro $(\alpha)$ a ser determinado, sua estreita faixa deval ores $(0,5$ a 1$)$ demonstrou ser possível a análise de uma ampla categoria de solos com boa representatividade. Evidentemente, o conhecimento preciso de $(\alpha)$ contribuirá para que as predições sejam ainda mais confiáveis.

3. Comparando os resultados obtidos do modelo da condutividade e os da curva experimental da condutividade de dois sol os: um L atossol o Vermel hoAmarelo e um Argissol o Amarelo, verificou-se que o modelo proposto mostrou-se simples em sua utilização, sendo capaz de predizer satisfatoriamente a condutividadehidráulica dos solos não saturados.

\section{LITE RATURA CITADA}

ANGULO-J ARAMILLO, R.; VANDERVAERE, J .P.; ROULIER, S.; THONY, J .L.; GAUDET, J .P. \& VAUCLIN, M. Field measurement of soil surface hydraulic properties by disc and ring infiltrometers: a review and recent devel opments. Soil Till. Res., 55:1-29, 2000.

BEAR, J. Dynamics of fluids in porous media. New York, Dover Publications, 1972. 764p.

BRADDOCK, R.D.; PARLANGE, J .-Y. \& LEE, H. Application of a soil water hysteresis model to simple water retention curves. Transp. Porous Media, 44:407-420, 2001.

BRAUD, I.; ANTONINO, A.C.D.; VAUCLIN, M.; THONY, L. \& RUELLE, P. A simple soil plant atmosphere transport model (SISPAT) development and field verification. J . Hydrol., 166:213-250, 1995.

BROOKS, R.H. \& COREY, A.T. Hydraulic properties of porous media. Hydrol. Fort Collins, Colo. State University, 1964. $27 p$.

BURDINE, N.T. Relative permeability calculation from size distribution data. Trans. AIME, 198:71-78, 1953.

CHILDS, E.C. \& COLLIS-GEORGE, N. The permeability of porous materials. Proc. Roy. Soc., Ser. A 201:392-405, 1950.

DULLIEN, F.A.L. Porous Media: Fluid transport and pore structure. New York, Academic Press, 1979. 306p.
FALLEIROS, M.C.; PORTEZAN, O.; OLIVEIRA, J.C.M.; BACCHI, O.O.S. \& REICHARDT, K. Spatial and temporal variability of soil hydraulic conductivity in relation to soil water distribution, using an exponential model. Soil Till. Res., 45:279-285, 1998.

FUENTES C. Approche fractale des transferts hydriques dans les sols non-saturés. Université J oseph Fourier de Grenoble, France, 1992. 267p. (Tese de Doutorado)

FUENTES, C.; VAUCLIN, M.; PARLANGE, J.-Y. \& HAVERKAMP, R. Soil-water conductivity of a fractal soil. En Fractals in Soil Science, Boca Raton, CRC press, 1998. p.333-340.

FUENTES, C.; BRAMBILA, F .; VAUCLIN, M.; PARLANGE, J .Y. \& HAVERKAMP, R. Modelación fractal de la conductividad hidráulica de los suelos no saturados. Ing. Hidráulica México, 16:119-137, 2001.

GUPTA, S.C. \& LARSON, W.E. Estimating soil water retention characteristics from particle-size distribution, organic matter percent and bulk density. Water Res. Res., 15:16331635, 1979.

HAVERKAMP, R. \& PARLANGE, J.-Y. Predicting the waterretention curvefrom particle-size distribution: I. Sandy soils without organic matter. Soil Sci., 142:325-339, 1986.

HILLEL, D.; KRENTOS, V.D. \& STYLLIANOU, Y. Procedure and test of an internal drainage method for measuring hydraulic characteristics "in situ". Soil Sci., 144:395-400, 1972.

KLUTE, A. \& DIRKSEN, C. Hydraulic conductivity and diffusivity: Laboratory methods. In: KLUTE A., ed., "Methods of soil analysis. Part 1: Physical and Mineralogical Methods." Madison, American Society of Agronomy, 1986.

LIBARDI, P.L.; REICHARDT, K.; NILSEN, D.R. \& BIGGAR, J.W. Simple field methods for estimating soil hydraulic conductivity. Soil Sci. Soc. Am. J ., 44:3-7, 1980.

MANDELBROT, B.B. The fractal geometry of nature. San Francisco. Freeman, 1983.

MUALEM, Y. A new model for predicting the hydraulic conductivity of unsaturated porous media. Water Res. Res., 12:513-522, 1976.

MUALEM, Y. \& DAGAN, G. Hydraulic conductivity of soils: unified approach to the statistical models. Soil Soil Sci. Soc. Am. J ., 42:392-395, 1978.

NETTO, A.M.; ANTONINO, A.C.D.; AUDRY, P.; CARNEIRO, C.J.G. \& DALL'OLIO, A. Condutividade hidráulica não saturada de um Podzólico Amarelo da zona da mata Norte de Pernambuco. Pesq. Agropec. Bras., 35:1221-1228, 2000.

OLESCHKO, K.; FUENTES, C.; BRAMBILA, F. \& ALVAREZ, R. Linear fractal analysis of threemexican soils in different management systems. Soil Technol., 10:207-223, 1997.

PURCELL, W.R. Capillary pressures - Their measurement using mercury and the calculation of permeability. Trans. AIME, 186:39, 1949.

RAWLS, W.J . \& BRAKENSIEK, D.L. Estimating soil water retention from soil properties. Am. Soc. Civ. Eng., 108:167171, 1981. 
REICHARDT, K. Capacidade decampo. R. Bras. Ci. Solo, 12:211216, 1988.

RIEU, M. \& SPOSITO G. Fractal fragmentation, soil porosity, and soil water properties: I. Theory. Soil Sci. Soc. Am. J., 55:1231-1238, 1991a.

RIEU, M. \& SPOSITO G. Fractal fragmentation, soil porosity, and soil water properties: II. Applications. Soil Sci. Soc. Am. J ., 55:1239-1244, 1991b.

SAXTON, K.E.; RAWLS, W.J .; ROMBERGER, J.S. \& PAPENDICK, R.I. Estimating generalized soil water characteristics from texture. Soil Sci. Soc. Am. J ., 50:10321036, 1986.

van GENUCHTEN, M.Th. A closed-form equation for predicting the hydraulic conductivity of unsaturated soils. Soil Sci. Soc. Am. J ., 44:892-898, 1980.

van DAM, J .C.; STRICKER, J .N.M. \& DROOGERS, P. I nverse method to determine soil hydraulic functions from multistep outflow experiments. Soil Sci. Soc. Am. J ., 58:647652, 1994.

WILLMOTT, C.J .; ACKLESON, S.G.; DAVIS, R.E.; FEDEMA, J .J .; KLINK, K.M.; LEGATES, D.R; O'CONNELL, J . \& ROWE, C.M. Statistics for the evaluation and comparison of models. J . Geophys. Res., 90:8995-9005, 1985.

ZHANG, R. \& van GENUCHTEN, M.Th. New models for unsaturated soil hydraulic properties. Soil Sci., 158:77-85, 1994.

\section{APÊ NDICE}

As integrais na equação 16 são todas do tipo: $\int \frac{\varepsilon^{\mathrm{s}-1} \mathrm{~d} \varepsilon}{|\psi|^{4 \mathrm{su}}}$

nas quais $u=\alpha$ ou $u=1-\alpha$. Os limites das integrais no numerador correspondema $\varepsilon=0$ e $\varepsilon=\theta$. No denominador, o limite inferior é $\varepsilon=0$ e o limite superior corresponde à porosidade total $\phi$. Desprezando a pequena fração dear presa nos poros, tem-se que $\varepsilon=\phi=\theta_{\mathrm{s}}$.

U sando a relação entre a porosi dade $(\varepsilon)$ e o grau de saturação (S), $\varepsilon=\theta_{S} S$, e sem perda de generalidade (no texto estão indicadas as modificações para introduzir no model o a umidade residual e obter os valores efetivos), resulta:

$$
\frac{1}{\left|\psi_{\mathrm{d}}\right|^{4 \mathrm{su}}} \int \frac{\varepsilon^{\mathrm{s}-1} \mathrm{~d} \varepsilon}{\left(\frac{\varepsilon}{\theta_{\mathrm{s}}}\right)^{-4 \mathrm{su} / \mathrm{mn}}\left[1-\left(\frac{\varepsilon}{\theta_{\mathrm{s}}}\right)^{1 / \mathrm{m}}\right]^{4 \mathrm{su} / \mathrm{n}}}
$$

Fazendo a substituição: $\varepsilon=\theta_{\mathrm{s}} \mathrm{x}^{\mathrm{m}}$ a integral simplifica-se em:

$$
\frac{\left(\theta_{\mathrm{S}}\right)^{\mathrm{s}} \mathrm{m}}{\left|\psi_{\mathrm{d}}\right|^{4 \mathrm{su}}} \int \mathrm{x}^{\mathrm{sm}+4 \mathrm{su} / \mathrm{n}-1}(1-\mathrm{x})^{-4 \mathrm{su} / \mathrm{n}} \mathrm{dx}
$$

Logo obtêm-se, para as integrais do numerador:

$$
\frac{\left(\theta_{\mathrm{S}}\right)^{\mathrm{s}} \mathrm{m}}{\left|\psi_{\mathrm{d}}\right|^{4 \mathrm{su}}} \int_{0}^{\mathrm{z}} \mathrm{x} \text { sm+4su/n-1}(1-\mathrm{x})^{-4 \mathrm{su} / \mathrm{n} \mathrm{dx}} ; \mathrm{z}=\left(\frac{\theta}{\theta_{\mathrm{S}}}\right)^{1 / \mathrm{m}}=\mathrm{S}_{\mathrm{e}} \mathrm{e}^{1 / \mathrm{m}}
$$

e para aquelas do denominador

$$
\frac{\left(\theta_{\mathrm{S}}\right)^{\mathrm{s} m}}{\left|\psi_{\mathrm{d}}\right|^{4 \mathrm{su}}} \int_{0}^{1} \mathrm{x}^{\mathrm{sm}+4 \mathrm{su} / \mathrm{n}-1}(1-\mathrm{x})^{-4 \mathrm{su} / \mathrm{n}} \mathrm{dx}
$$

Reconhecem-se imediatamente estas integrais como a função beta incompleta:

$$
\left.\beta_{I}(z, p, q)=\int_{0}^{z} x^{p-1}(1-x)\right)^{q-1} d x
$$

e a função beta completa

$$
\beta(\mathrm{p}, \mathrm{q})=\int_{0}^{1} \mathrm{x}^{\mathrm{p}-1}(1-\mathrm{x})^{\mathrm{q}-1} \mathrm{dx}=\frac{\Gamma(\mathrm{p}) \Gamma(\mathrm{q})}{\Gamma(\mathrm{p}+\mathrm{q})}
$$

na qual $\Gamma(\cdot)$ éa função gama de Euler. Estas funções convergem sempre que $p>0$ e $q>0$.

A equação 16 torna-se final mente:

$$
\frac{\mathrm{K}\left(\mathrm{S}_{\mathrm{e}}\right)}{\mathrm{K}_{\mathrm{S}}}=\frac{\Gamma(\mathrm{p}+\mathrm{q}) \Gamma\left(\mathrm{p}^{\prime}+\mathrm{q}^{\prime}\right)}{\Gamma(\mathrm{p}) \Gamma(\mathrm{q}) \Gamma\left(\mathrm{p}^{\prime}\right) \Gamma\left(\mathrm{q}^{\prime}\right)} \beta_{\mathrm{I}}\left(\mathrm{S}_{\mathrm{e}}^{1 / \mathrm{m}}, \mathrm{p}, \mathrm{q}\right) \beta_{\mathrm{I}}\left(\mathrm{S}_{\mathrm{e}}^{\left.1 / \mathrm{m}, \mathrm{p}^{\prime}, \mathrm{q}^{\prime}\right)}\right.
$$

com o seguinte conjunto de valores:

$$
\mathrm{p}=\mathrm{sm}+\frac{4 \mathrm{~s} \alpha}{\mathrm{n}}, \mathrm{q}=1-\frac{4 \mathrm{~s} \alpha}{\mathrm{n}}, \mathrm{p}^{\prime}=\mathrm{sm}+\frac{4 \mathrm{~s}(1-\alpha)}{\mathrm{n}} \text { e } \mathrm{q}^{\prime}=1-\frac{4 \mathrm{~s}(1-\alpha)}{\mathrm{n}}
$$

Daí, podem-se obter os dois casos limites:

a) Média Geométrica $\left(\alpha={ }^{1} / 2\right): p=p^{\prime}=s m+\frac{2 s}{n}$ e q $=q^{\prime}=1-\frac{2 s}{n}$

b) PoroNeutro $(\alpha=1): p=s m+\frac{4 s}{n}, p^{\prime}=s m, q=1-\frac{4 s}{n} e_{q^{\prime}}=1$

Neste ponto, é necessário verificar as condições de convergência das soluções. Para um problema fisicamente bem posto (aquele em que $\mu \leq \phi$ ), é possível mostrar que o índice fractal s dado pela equação 7 apresenta solução única no intervalo $1 / 2<\mathrm{s}<1$. Como os parâmetros de forma estão limitados a $m>0$ e $n>0$, segue-se então que:

a) Média Geométrica: $p>0, p^{\prime}>0$, e, para seter $q>0$ e $q^{\prime}>0$, basta que $n>2 s$.

b) PoroNeutro: $p>0, p^{\prime}>0, q^{\prime}=1>0$, e, para seter $q>0$, basta que $n>4 s$.

Nestas condições, a convergência estará assegurada. É interessante investigar as soluções para as quais a função beta apresenta uma forma analítica, sendo as mais simples aquelas para as quais $p=1$.

a) Média Geométrica:

$$
\frac{\mathrm{K}\left(\mathrm{S}_{\mathrm{e}}\right)}{\mathrm{K}_{\mathrm{S}}}=\left[\frac{\Gamma(1+\mathrm{q})}{\Gamma(1) \Gamma(\mathrm{q})} \beta_{\mathrm{I}}\left(\mathrm{S}_{\mathrm{e}}^{1 / \mathrm{m}}, 1, \mathrm{q}\right)\right]^{2}
$$




$$
\begin{gathered}
\frac{\mathrm{K}\left(\mathrm{S}_{\mathrm{e}}\right)}{\mathrm{K}_{\mathrm{S}}}=\left\{\frac{\mathrm{q} \Gamma(\mathrm{q})}{\Gamma(1) \Gamma(\mathrm{q})} \frac{1}{\mathrm{q}}\left[1-\left(1-\mathrm{S}_{\mathrm{e}}^{1 / \mathrm{m}}\right)\right]^{\mathrm{q}}\right\}^{2} \\
\mathrm{~K}\left(\mathrm{~S}_{\mathrm{e}}\right)=\mathrm{K}_{\mathrm{S}}\left[1-\left(1-\mathrm{S}_{\mathrm{e}}^{1 / \mathrm{m}}\right)^{1-2 \mathrm{~s} / \mathrm{n}}\right]^{2} \operatorname{com} \mathrm{n}=\frac{2 \mathrm{~s}}{1-\mathrm{sm}}
\end{gathered}
$$

b) Poro Neutro:

$$
\frac{\mathrm{K}\left(\mathrm{S}_{\mathrm{e}}\right)}{\mathrm{K}_{\mathrm{S}}}=\frac{\Gamma(1+\mathrm{q}) \Gamma\left(\mathrm{p}^{\prime}+1\right)}{\Gamma(1) \Gamma(\mathrm{q}) \Gamma\left(\mathrm{p}^{\prime}\right) \Gamma(1)} \beta_{\mathrm{I}}\left(\mathrm{S}_{\mathrm{e}}^{1 / \mathrm{m}}, 1, \mathrm{q}\right) \beta_{\mathrm{I}}\left(\mathrm{S}_{\mathrm{e}}^{1 / \mathrm{m}}, \mathrm{p}^{\prime}, 1\right)
$$

$$
\begin{gathered}
\frac{\mathrm{K}\left(\mathrm{S}_{\mathrm{e}}\right)}{\mathrm{K}_{\mathrm{S}}}=\frac{\mathrm{q} \Gamma(\mathrm{q}) \mathrm{p}^{\prime} \Gamma\left(\mathrm{p}^{\prime}\right)}{\Gamma(1) \Gamma(\mathrm{q}) \Gamma\left(\mathrm{p}^{\prime}\right) \Gamma(1)} \frac{1}{\mathrm{q}}\left[1-\left(1-\mathrm{S}_{\mathrm{e}}^{1 / \mathrm{m}}\right)^{\mathrm{q}}\right] \frac{1}{\mathrm{p}^{\prime}}\left(\mathrm{S}_{\mathrm{e}}^{1 / \mathrm{m}}\right)^{\mathrm{p}^{\prime}} \\
\mathrm{K}\left(\mathrm{S}_{\mathrm{e}}\right)=\mathrm{K}_{\mathrm{S}}\left(\mathrm{S}_{\mathrm{e}}\right)^{\mathrm{s}}\left[1-\left(1-\mathrm{S}_{\mathrm{e}}^{1 / \mathrm{m}}\right)^{1-4 \mathrm{~s} / \mathrm{n}}\right] \operatorname{com} \mathrm{n}=\frac{4 \mathrm{~s}}{1-\mathrm{sm}}
\end{gathered}
$$

Por outro lado, o fato de que $\mathrm{n}>0$, exige que sm $<1$ em ambos os modelos, ou de forma equivalente, e já incorporando as outras restrições acima sobre $n$, chega-se a $0<\mathrm{sm}<1$. 